\title{
Time Resolved Growth of Pt From Single Atoms to Nanocrystals With HR-TEM
}

\author{
Peter Ercius $^{1}$, Yuri Borodko ${ }^{2}$ and Gabor Somorjai ${ }^{2,3}$ \\ 1. National Center for Electron Microscopy, Lawrence Berkeley National Laboratory, Berkeley, USA \\ 2. Materials Science Division, Lawrence Berkeley National Laboratory, Berkeley, USA \\ 2. Department of Chemistry, UC Berkeley, Berkeley, USA
}

$\mathrm{Pt}$ is used in a wide range of practical applications for catalysis, biomedicine and electronics. The dynamics of structural transformations of Pt aggregates in the "quantum size" range where their molecular structure transforms into crystalline nanoparticles with metallic properties is an important issue in nanoscience. The size range for the use of $\mathrm{Pt}$ extends from mononuclear compounds for heterogeneous catalysis to bulk metal for homogenous catalysis. At the nanoscale, $2-10 \mathrm{~nm}$ diameter nanoparticles have been discovered as new selective catalysts and are under extensive study. A difficult size range to study are small metal clusters with a molecular structure containing $\sim 5-30$ atoms that have not yet formed into solid crystals. Numerous of these $\mathrm{Pt}_{\mathrm{n}}$ clusters (such as Pt-blue) have been synthesized and exhibit a variety of structures and oxidation states as measured in large crystallized ensembles by x-ray crystallography. [1] Polymeric macroligands such as the Poly(amido amine) (PAMAM) dendrimer stabilize Pt aggregates, and Pt-PAMAM are used as active catalysts in liquidphase reactions. We use aberration-corrected high-resolution TEM to directly image Pt-PAMAM aggregates and Pt cluster coalescence under high-dose TEM conditions. [2]

Photoreduction of Pt mononuclear compounds results in aggregation of the species, but the PAMAM dendrimer acts as a "cage" to contain Pt atoms from diffusing out of the dendrimer during photoreduction with $254 \mathrm{~nm}$ UV light (Figure 1). The reduced Pt atoms coalesce inside the dendrimer, which limits their size to $\sim 1 / 2$ the size of the dendrimer as measured by resonance UV ramen after 360 minutes of irradiation. [2] The intermediate stages of growth are of great interest and require direct imaging techniques to investigate.

We use the aberration corrected TEAM I instrument at NCEM to image single Pt atoms, Pt clusters and Pt nanoparticles grown in PAMAM dendrimer with graphene as the substrate. The instrument provides sufficient resolving power and contrast to directly image single $\mathrm{Pt}$ atoms with $\sim 1 \mathrm{~s}$ acquisition time which allows time resolved movies of Pt-Pt interactions. We used $80 \mathrm{keV}$ with a $-10 \mu \mathrm{m}$ spherical aberration such that atoms look bright at under focus. The clusters were first reduced under UV light for different times in solution to produce clusters of different sizes. Figure 2a shows mononuclear Pt ions and little change is evident after $30 \mathrm{~s}$ of high irradiation (Figure 2b). Figure 2c shows Pt-blue-like linear clusters, and Figure $2 \mathrm{~d}$ shows a Pt nanocrystal. It is believed that the mononuclear Pt atoms first form linear chains, which are the building blocks of crystals.

The mechanism by which disordered aggregates transform into well-ordered Pt nanocrystals is a matter of debate, but we believe that the number of atoms contained within the aggregate plays a critical role in this transformation. Our study shows that during crystal growth, Pt atoms may self-assemble into kinetically metastable intermediates, referred to as mesocrystals, superstructures with crystal-like appearance. [3] Such mesocrystals consist of Pt-blue building blocks, which are oriented and interspaced by amidate ligands. Electron beam irradiation $\left(10^{3} \mathrm{e}^{-} / \AA^{2} \mathrm{~s}\right)$ strongly reduces the Pt aggregates and also causes them to change under the beam. Figure 3 shows selected images from 3 movies arranged in 
columns with 1.5 second time resolution. Figure 3a shows a small collection of atoms - just below the disordered-to-crystalline transition size - transform reversibly from a disordered aggregate to a mesocrystal, which is stable for $3 \pm 1.5 \mathrm{~s}$. Coalescence events of two aggregates into a single crystal (Figure $3 \mathrm{~b}$ ) and oriented attachment of an aggregate to a crystal (Figure 3c) are also shown. We believe that the mechanism of Pt coalescence occurs by the same process whether the reducing irradiation is UV light or an electron beam, and the dynamics observed in this study provide insight into the transformation of Pt ions to nanocrystals.

\section{References:}

[1] JK Barton, HN Rabinowitz, DJ Szalda and SJ Lippard, Journal of the American Chemical Society 99 (1977) pp. 2827- 2829.

[2] Y Borodko, P Ercius, V Pushkarev, C Thompson and G Somorjai, Journal of Physical Chemistry Letters, 3 (2012) pp. 236 - 241.

[3] M Niederberger and H Colfen, Physical Chemistry Chemical Physics 8 (2006) pp. 3271 - 3287.

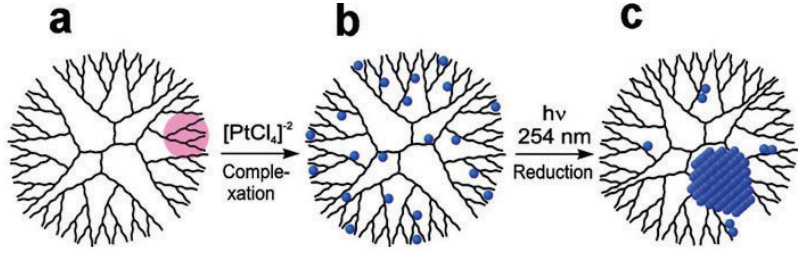

Figure 1. The Pt-PAMAM macroligand during complexation and UV irradiation from Pt mononuclear compounds to nanocrystals.
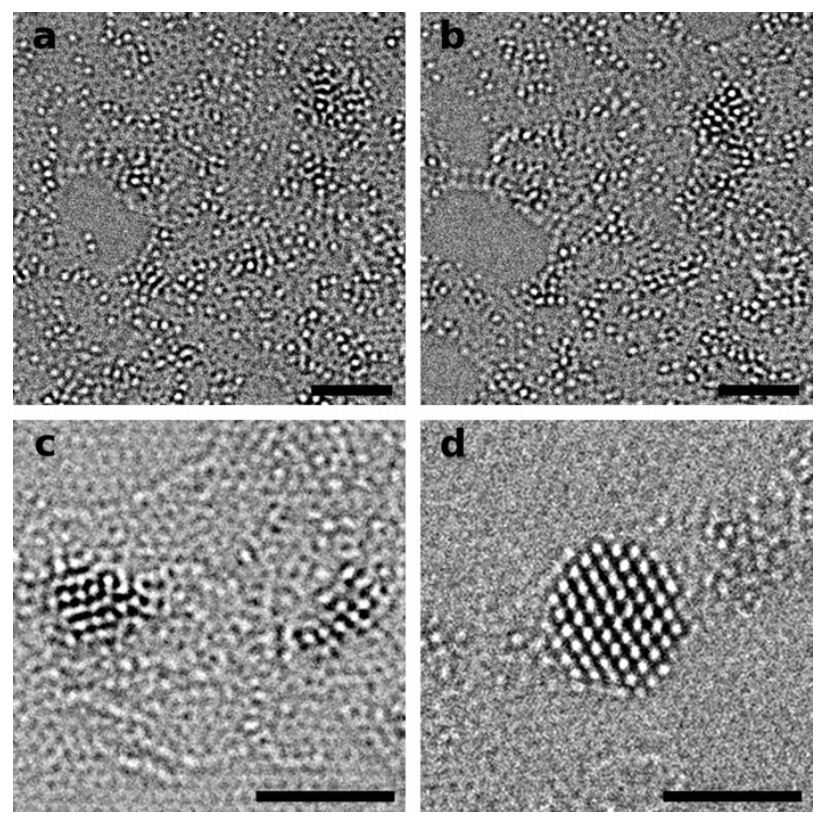

Figure 2. (a-b) HR-TEM images of Pt atoms (white) on graphene (a) before and (b) after $30 \mathrm{~s}$ of electron beam irradiation. (c) Intermediate linear Pt-blue like compounds. (d) A Pt nanocrystal. $2 \mathrm{~nm}$ scale bars
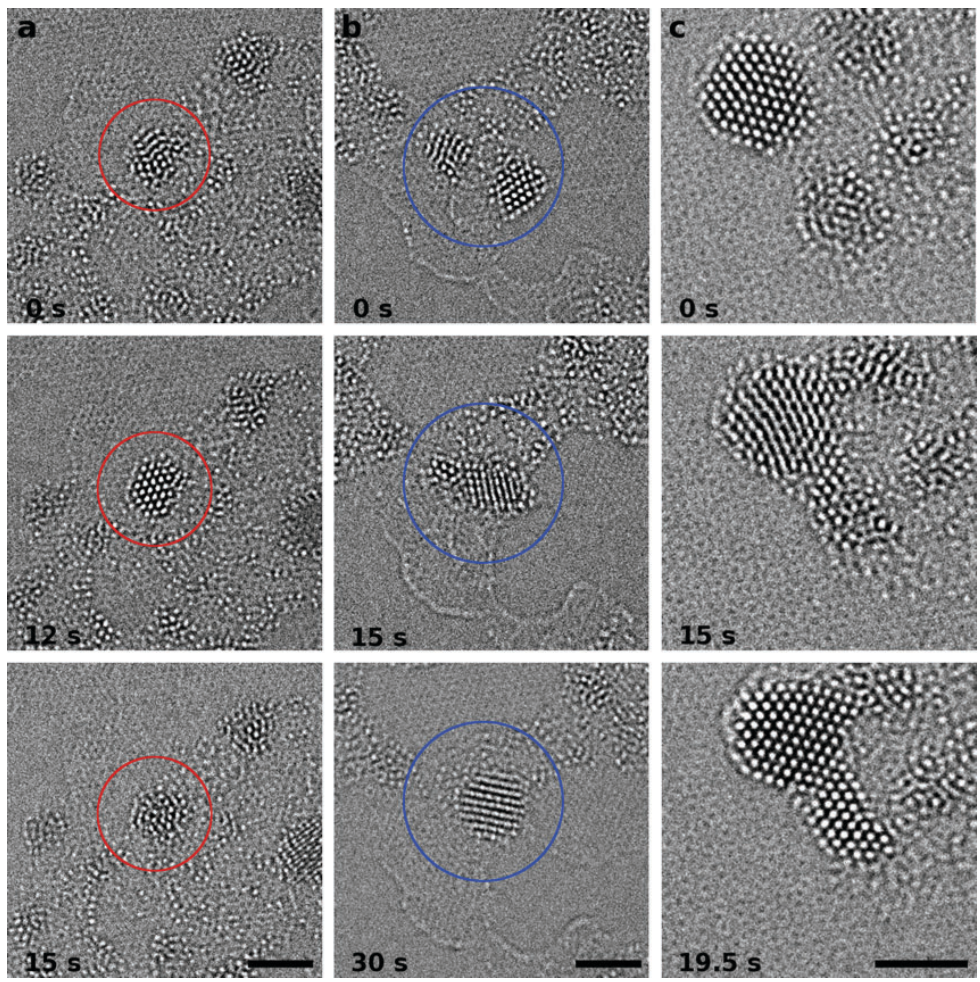

Figure 3. Images extracted from 3 time series with 1.5 $\mathrm{s}$ time resolution of $\mathrm{Pt}$ atoms (white dots), aggregates and crystals. (a) An aggregate (red circle) reversibly transforms between a disorderd aggregate and a mesocrystal. (b) Two aggregates (blue circle) combine to form a single crystal. (c) Oriented attachment of an aggregate with a larger crystal. $2 \mathrm{~nm}$ scale bars. 